\title{
Étude par coulométrie de la pénétration de l'eau dans des films de polymères formulés
}

\author{
K. Vallée-Rehel ${ }^{1, *}$, B. Mariette ${ }^{1}$, P.A. Hoarau ${ }^{1}$ et P. Guerin ${ }^{2}$ \\ ${ }^{1}$ DCN Indret, CESMAN, 44620 La Montagne, France \\ ${ }^{2}$ Laboratoire de Physico-Chimie des Biopolymères, Université de Paris XII-UMR 27 CNRS, 94320 Thiais, France

\begin{abstract}
Hulls protection towards settlement and growth of fouling is obtained by antifouling paints with a progressive release of toxic molecules at the ships surface. The research of a new generation of paints more compatible with living species implies to understand the phenomena involved in paints erosion and biocides release. Water penetration in polymeric matrix is important in regard to the control of ester functions hydrolysis and so to the control of bioactive molecules release. Among the different analytical methods aimed at the determination of the water amount in polymers, we have proposed to use coulometry in order to study the water penetration in polymeric films immersed in synthetic sea water. The analytical protocols de veloped have enabled to understand the evolution of six different matrixes during the immersion and the comparison of their ability to control release. Coulometry has made possible the study of the effects of additives used in the paints formulation on water uptake. Experimental data have shown that additives modify the evolution of the binder during immersion and that activity of paints is complex. Coulometry is a useful method to test polymers aimed at marine applications.
\end{abstract}

Key words. coulometry - water uptake - antifouling - paints - DSC - TGA.

\section{Introduction}

L'eau diffusant dans une peinture provoque son gonflement [1] et peut entraîner une perte d'adhésion, une détérioration des propriétés mécaniques ou un début de corrosion [2] du support. Mais, dans le cas des peintures antisalissures, elle est essentielle en regard de la protection recherchée par solubilisation et libération de molécules bioactives. Le phénomène de pénétration d'eau dans les films de peintures antisalissures reste encore peu étudié bien qu'il soit au centre de l'hydrolyse, de l'érosion de la peinture et donc de la libération du biocide. Le classement des liants et des charges utilisés dans les formulations selon leur hydrophobie/hydrophilie tient compte avant tout de leur structure chimique, de leur solubilisation dans l'eau et les solvants organiques classiques et prend rarement en compte les mélanges complexes dus à la formulation. Pour une plus grande efficacité de ces peintures et une meilleure compréhension de leur fonctionnement, il est important de mettre en place un protocole de tests analytiques. Notre projet a pour objet de rechercher et de développer des peintures antisalissures biodégradables et d'établir un protocole de tests analytiques.

Différentes techniques analytiques permettent de quantifier et de localiser l'eau présente dans le film [3,4]. La gravimétrie peut être utilisée mais elle est souvent délicate à mettre en œuvre car des phénomènes d'extraction de petites molécules présentes dans le film (plastifiants, additifs, inhibiteurs...) se superposent à la diffusion de l'eau. La mesure de l'impédance électrochimique tend à se développer car elle apporte des informations plus précises sur la pénétration de l'eau dans un revêtement appliqué sur un support métallique [5-10]. Lorsque la détermination de la quantité d'eau présente n'est pas suffisante et que l'on souhaite obtenir des informations sur la distribution de l'eau dans les polymères, il est possible d'utiliser la micrographie RX ou l'imagerie RMN [11-13].

Dans cette étude, nous proposons d'utiliser une autre méthode de quantification de l'eau absorbée : la coulométrie. Le protocole expérimental proposé permet de suivre le comportement de polymères acryliques au cours de l'immersion en eau saline durant une période d'un mois. Notre objectif est de mettre en place une méthode analytique fiable permettant d'apprécier précisément le caractère hydrophobe/hydrophile d'un liant en vue de son incorporation dans une peinture antisalissures. L'influence de trois charges toxiques (biocides) à base de cuivre utilisées dans les peintures est également étudiée de façon à maîtriser la formulation dans sa globalité.

\section{Partie expérimentale}

\section{Matériaux}

Les produits étudiés étant commerciaux, leurs formules chimiques ne peuvent pas être divulguées systématiquement. Les exemples donnés sont représentatifs des molécules réellement utilisées.

\section{Les polymères étudiés sont :}

1) le BIOMET ${ }^{\circledR}$ est utilisé dans les peintures antisalissures autopolissantes. Il s'agit d'un produit commercial (fournisseur : ELF ATOCHEM). Généralement, les liants organostanniques pour peintures antisalissures sont des co- ou terpolymères associant des unités méthacrylate de méthyle (25\% molaire, par exemple), acrylate de butyle (10\% molaire, par exemple) et méthacrylate de tributylétain (65\% molaire, par exemple). Les polymères

* Correspondance et tirés à part : Université de Bretagne Sud, 4 rue Jean ZAY, F-56325 Lorient, France.

Reçu le 13 mai 1997 ; révisé le 29 octobre 1997 ; accepté le 03 décembre 1997. 
organostanniques possèdent la caractéristique unique de subir une hydrolyse de la liaison ester latérale à $\mathrm{pH}$ voisin de 8 ( $\mathrm{pH}$ de l'eau de mer) et peuvent ainsi libérer des ions stanniques biocides de certains organismes et microorganismes marins. Les peintures correspondantes possèdent une efficacité exemplaire sur une durée pouvant atteindre 4 à 5 ans. Toutefois leur toxicité envers le milieu marin conduit à une restriction grandissante de leur utilisation. Dans notre étude, il s'agit de produits de référence tant au niveau de l'efficacité que de leur mode d'action (Fig. 1).
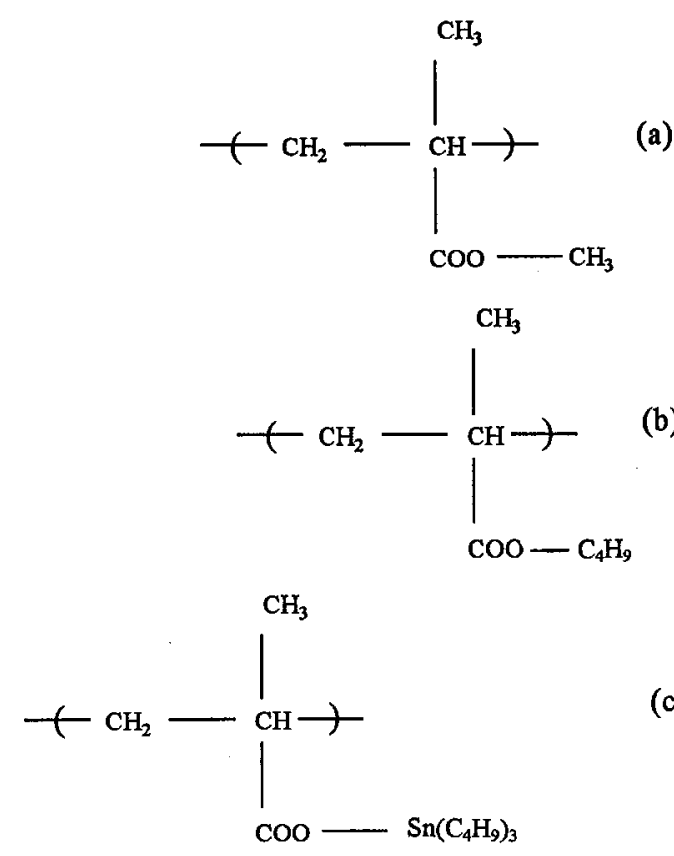

Fig. 1. Composés entrant dans la composition du BIOMET : (a) méthacrylate de méthyle, (b) acrylate de butyle et (c) méthacrylate de tributylétain.

2) la DERAKANE ${ }^{\circledR}$ est une résine vinylester obtenue par polymérisation du mélange à base de Derakane 411-00 et de styrène. La Derakane 411-00 (fournisseur : DOW CHEMICALS) est une résine diméthacrylique à base de diglycédyléther de bisphénol A. Cette résine est connue pour sa résistance à l'hydrolyse et son hydrophobie. Il s'agit d'un matériau hydrophobe de référence (Fig. 2).

3) les résines $\mathbf{A}, \mathbf{B}$ et $\mathbf{C}$ sont des polymères acryliques présentant des caractéristiques d'hydrophilie/hydrophobie différentes. En effet, ces résines associent dans des proportions variables des unités « hydrophobes » (méthacrylate de méthyle, par exemple), « hydrophiles » (acide méthacrylique, par exemple) et « hydrolysables » (méthacrylate de benzoxyéthyle, par exemple) (cf. Fig. 3). Il s'agit d'une nouvelle génération de liants pour peintures antisalissures sans dérivés de l'étain et respectant davantage l'environnement. L'activité antisalissures des peintures correspondantes est obtenue par libération continue de molécules biocides grâce à une dégradation hydrolytique ou physique du liant.

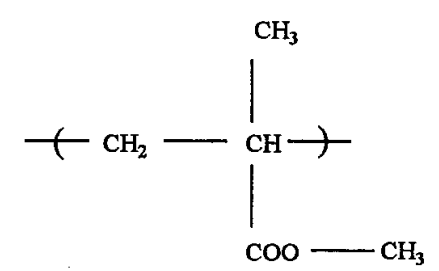

(a)

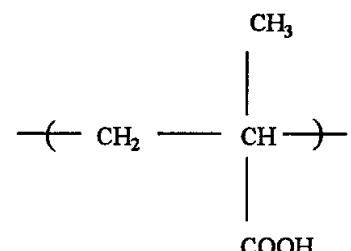

(b)

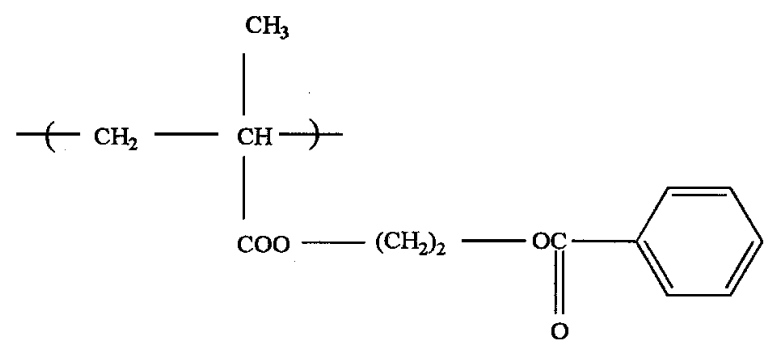

(c)

Fig. 3. Structure chimique de l'unité de répétition des polymères utilisés : (a) méthacrylate de méthyle, (b) acide méthacrylique et (c) méthacrylate de benzoxyéthyle.

Les caractères « hydrophobe », « hydrophile » et « hydrolysable» sont attribués aux unités en fonction de leur structure chimique et des données de la littérature concernant leur comportement à l'hydrolyse. Le méthacrylate de méthyle (MAM) est reconnu « hydrophobe » en raison de la présence du groupement méthyle sur la chaîne latérale. L'acide méthacrylique (AM) est « hydrophile » en raison de la présence du groupement $\mathrm{COOH}$ sur la chaîne latérale. Le méthacrylate de benzoxyéthyle (BEMA) est « hydrolysable » en raison de la structure chimique du groupement ester favorisant une réaction d'hydrolyse sur la chaîne latérale. L'étude du comportement des polymères $\mathrm{A}$, $\mathrm{B}, \mathrm{C}$ en immersion nous permettra de confirmer ou non ce classement.

La résine $\mathbf{A}$ est un homopolymère et ne possède que des unités « hydrophobes » du type méthacrylate de méthyle. La résine $\mathbf{B}$ est un copolymère associant des unités « hydrophobes » (par exemple, méthacrylate de méthyle à $70 \%$ molaire) et des unités « hydrophiles » (par exemple, acide méthacrylique à $30 \%$ molaire). La résine $\mathbf{C}$ est un terpolymère associant des unités « hydrophobes » (méthacrylate de méthyle à $20 \%$ molaire), « hydrophiles » (acide méthacrylique à $30 \%$ molaire) et « hydrolysables » (méthacrylate de benzoxyéthyle à $50 \%$ molaire).

Les polymères sont obtenus par polymérisation radicalaire en solution (dioxane anhydre) amorcée par l'azobisisobutyronitrile $(\mathrm{AIBN})$ à $70{ }^{\circ} \mathrm{C}$. Une étude de la réactivité des différents comonomères a montré que des polymères

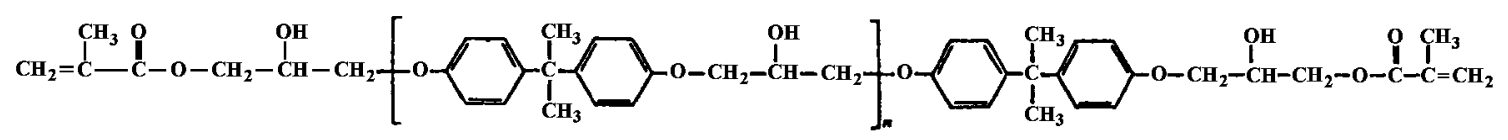

Fig. 2. Structure chimique de la Derakane. 
statistiques sont obtenus. Les masses molaires moyennes déterminées par chromatographie d'exclusion stérique (CES) (étalonnage simple avec standard polystyrène, THF) sont rassemblées dans le tableau I :

Tableau I. Masses molaires moyennes des résines A, B, C déterminées par CES.

\begin{tabular}{lccc}
\hline Résine & $\bar{M}_{n}$ & $\bar{M}_{p}$ & $I$ \\
\hline A & 23000 & 50000 & 2,2 \\
B & 11000 & 21000 & 1,8 \\
C & 8000 & 19000 & 2,3 \\
\hline
\end{tabular}

$M_{\mathrm{n}}$ : moyenne molaire moyenne en nombre,

$M_{\mathrm{p}}:$ masse molaire moyenne en poids,

$I$ : indice de polydispersité $\left(I=M_{\mathrm{p}} M_{\mathrm{n}}\right)$.

4) la colophane, résine naturelle constituée à $90-95 \%$ d'acides résiniques et de $5-10 \%$ de produits insaponifiables. Hydrosoluble, la colophane est incorporée dans les peintures pour favoriser la pénétration de l'eau et la libération des biocides. L'utilisation de la colophane comme liant dans les peintures pose des difficultés à cause des mauvaises propriétés mécaniques et de la trop grande solubilité de ces peintures dans le milieu naturel (Fig. 4).

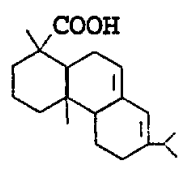
abiétique
Acide

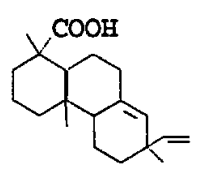

Acide dextropimarique

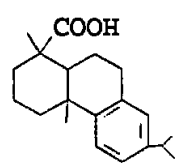

Acide déhydroabiétique
Fig. 4. Acides abiétiques présents dans la colophane.

Parallèlement à l'étude des résines non chargées, l'effet sur la pénétration d'eau de produits toxiques couramment utilisés a été étudié. Les charges utilisées et leurs proportions sont rassemblées dans le tableau II :

Tableau II. Charges utilisées.

\begin{tabular}{lc}
\hline Charges et additifs & Pourcentage massique incorporé (\%) \\
\hline $\begin{array}{l}\text { oxyde de cuivre } \\
\left(\mathrm{Cu}_{2} \mathrm{O}\right)\end{array}$ & 18 \\
$\begin{array}{l}\text { oxyde de cuivre modifié } \\
\left.\left(\mathrm{Cu} \mathrm{O}_{2} \mathrm{O}\right) \text { modifié }\right)\end{array}$ & 18 \\
$\begin{array}{l}\text { thiocyanate de cuivre } \\
(\mathrm{CuSCN})\end{array}$ & 3 \\
$\begin{array}{l}\text { tributyl étain } \\
(\mathrm{TBT})\end{array}$ & 3 \\
\end{tabular}

Ces biocides à base de cuivre sont des constituants classiques des formulations de peintures antisalissures : oxyde de cuivre rouge $\left(\mathrm{Cu}_{2} \mathrm{O}\right)$, oxyde de cuivre modifié (ayant subi un traitement de surface consistant en un enrobage à base d'un polymère non précisé pour améliorer la mouillabilité et atténuer la couleur rouge sombre) et thiocyanate de cuivre $(\mathrm{CuSCN})$. Le tributylétain (TBT) est utilisé comme molécule toxique modèle en raison de sa solubilité dans l'eau et de son activité biocide. L'ensemble des caractéristiques de ces charges (formule, pureté, granulométrie, fournisseur...) est rassemblé dans le tableau III.

Tableau III. Caractéristiques des charges utilisées.

\begin{tabular}{lcccc}
\hline Charge & Formule & Fournisseur & Pureté Granulométrie \\
\hline $\mathrm{Cu}_{2} \mathrm{O}$ & $\mathrm{Cu}_{2} \mathrm{O}$ & American Chemet & $>99$ & Hegman : 6 \\
$\mathrm{Cu}_{2} \mathrm{O}$ modifié & $\mathrm{Cu}_{2} \mathrm{O}$ & American Chemet & $>97,5$ & Hegman : 4 \\
$\mathrm{CuSCN}$ & $\mathrm{CuSCN}$ & Bardyke Chemicals & $>99$ & $15-25$ microns \\
TBT & $\mathrm{Sn}\left(\mathrm{C}_{4} \mathrm{H}_{9}\right)_{3}$ & Elf Atochem & $>99$ & $10-15$ microns \\
\hline
\end{tabular}

\section{Préparation des échantillons}

Les polymères sont solubilisés à $50 \%$ en masse dans un solvant organique, le xylène. Lors de la fabrication des vernis chargés, les additifs sont dispersés sous agitation. Les vernis sont appliqués sur des supports en matériau composite (résine vinylester-renfort fibres de verre) de dimension ( $\mathrm{mm}$ $\mathrm{L}, 1$, e) $80 \times 40 \times 5$. Après séchage à température ambiante, les plaques sont plongées dans l'eau saline normalisée (NF J 17087-Mars 1975, cf. Fig. 5), non agitée à température ambiante. Dans le cas de la résine DERAKANE ${ }^{\circledR}$, des plaques de résine non chargée, non renforcée, sont réalisées. Après polymérisation, les éprouvettes sont placées en immersion.

$\begin{array}{ll}\text { eau } & 20 \mathrm{~kg} \\ \mathrm{NaCl} & 600 \mathrm{~g} \\ \mathrm{H}_{3} \mathrm{BO}_{3} & 25 \mathrm{~g} \\ \mathrm{Na}_{2} \mathrm{HPO}_{4} & 3,8 \mathrm{~g} \\ \mathrm{Na}_{2} \mathrm{CO}_{3} & 20 \mathrm{~g}\end{array}$

Fig. 5. Composition de l'eau saline.

\section{Conditions de prélèvements}

A la sortie du milieu de vieillissement, la surface de la plaque est recouverte de gouttelettes d'eau. La difficulté de ce titrage est de mesurer uniquement l'eau contenue à l'intérieur du film et non l'eau résiduelle de surface. C'est pourquoi, la méthode adoptée pour prélever l'échantillon est la suivante. La plaque émergée est séchée à l'étuve à $23^{\circ} \mathrm{C}$, à $50 \%$ d'humidité relative, durant un temps $t$ variable. Ce temps correspond au moment de la disparition des dernières gouttelettes d'eau de la surface de la peinture. Un prélèvement, sur toute l'épaisseur du film, est effectué au cutter. Entre chaque prélèvement, la plaque est conservée dans un pot hermétiquement fermé, dans une salle climatisée à $20{ }^{\circ} \mathrm{C}$. Cinq analyses sont réalisées pour chacun des échantillons. 


\section{Appareillage}

Coulométrie : La coulométrie permet de doser l'eau dans les revêtements selon le titrage de Karl-Fischer. Dans le cadre de notre étude, pour nous affranchir des problèmes dus à l'utilisation de solvants, nous avons utilisé une méthode de dosage indirect nécessitant l'emploi d'un four. L'appareil utilisé est du type Coulomètre KF DL 37 (Mettler). Un «blanc» est systématiquement réalisé avec balayage d'azote sec dans le four et bullage dans la cellule de titrage. Les réactifs utilisés sont du type Hydranal : anolyte Hydranal Colomat A $(100 \mathrm{~mL})$ et catholyte Hydranal Colomat C $(5 \mathrm{~mL})$. Les masses de vernis analysées sont de l'ordre de $40 \mathrm{mg}$.

Analyse enthalpique différentielle (DSC) : La température de transition vitreuse $\left(T_{\mathrm{v}}\right)$ a été déterminée sur une prise de $10 \mathrm{mg}$. L'analyse a été réalisée sur une gamme de température allant de 0 à $150{ }^{\circ} \mathrm{C}$ à raison de $10^{\circ} \mathrm{C} /$ minute. L'appareil utilisé est du type DSC-7 (Perkin Elmer).

Analyse thermogravimétrique (TGA) : La température de début de dégradation a été déterminée sur une prise de $25 \mathrm{mg}$. L'analyse a été réalisée sur une gamme de température allant de 25 à $500{ }^{\circ} \mathrm{C}$ à raison de $10{ }^{\circ} \mathrm{C} /$ minute. L'appareil utilisé est du type TGA-7 (Perkin Elmer).

Spectrométrie de photoélectrons sous bombardement de rayons $X$ (ESCA) : L'appareil utilisé est un spectromètre LHS 12 Leybold. Deux types d'échantillons ont été analysés : la surface brute obtenue après séchage du vernis à l'air et la surface obtenue après érosion mécanique du vernis (obtention d'informations sur la composition « à cœur»). Les raies observées correspondent aux niveaux électroniques $1 \mathrm{~s}$ du carbone et de l'oxygène (cf. Tab. IV).

Tableau IV. Conditions d'analyse utilisées en ESCA.

\begin{tabular}{lc}
\hline Élément & Domaine d'énergie $(\mathrm{eV})$ \\
\hline $\mathrm{C}$ & $280-295$ \\
$\mathrm{O}$ & $532-538$ \\
\hline
\end{tabular}

Couplage chromatographie en phase gazeuse-spectromé trie de masse : colonne Hewlett-Packard FFAP phase réticulée à base de polyéthylèneglycol, chromatographe Hewlett-Packard 5890 series II, gaz vecteur Helium, débit $0,9 \mathrm{~mL} / \mathrm{mn}$, température initiale $60{ }^{\circ} \mathrm{C}$, température finale $200{ }^{\circ} \mathrm{C}$, vitesse de montée en température $10^{\circ} \mathrm{C} / \mathrm{mn}$, volume injecté $10 \mu \mathrm{L}$, spectromètre de masse Hewlett-Packard 5989A.

Chromatographie d'exclusion stérique (CES) : concentration massique des échantillons $2 \%$, solvant et phase mobile THF grade HPLC filtré et dégazé, débit $1 \mathrm{~mL} / \mathrm{mn}$, colonnes $\mu$-styragel $10^{4}, 10^{3}$ et 500 Angströms en série, réfractomètre différentiel Waters410, volume injecté $10 \mu \mathrm{L}$, étalons polystyrène isomoléculaires de masse variant de 5050 à 200000.

\section{Résultats et discussion}

\section{Vernis non chargés}

Lors de la mesure par coulométrie, l'eau contenue dans l'échantillon est désorbée dans le four sous l'effet de la cha- leur et est entraînée par un gaz de balayage inerte sec (azote) dans la cellule de titrage où elle est quantifiée. Le choix de la température du four consiste à réaliser un compromis entre la température permettant la libération totale de l'eau et la température de début de dégradation du polymère. C'est pourquoi, dans un premier temps, les différents polymères testés ont été soumis à des analyses thermiques.

Leurs températures de transition vitreuse ont été déterminées par analyse enthalpique différentielle (DSC) (cf. Tab. V) :

Tableau V. Températures de transition vitreuse des polymères étudiés.

\begin{tabular}{lc}
\hline Polymère & $T_{v}\left( \pm 3^{\circ} \mathrm{C}\right)$ \\
\hline BIOMET & 29 \\
RÉSINE A & 25 \\
RÉSINE A + COLOPHANE & 36 \\
RÉSINE B & 26 \\
RÉSINE C & 31 \\
DÉRAKANE & 107 \\
\hline
\end{tabular}

L'ensemble des polymères acryliques présentent des températures de transition vitreuse de l'ordre de $25-30^{\circ} \mathrm{C}$ nettement inférieures à celle du polymère vinylester DERAKANE $\left(T_{\mathrm{v}}=107{ }^{\circ} \mathrm{C}\right)$.

L'analyse thermique différentielle (TGA) a permis de déterminer les températures de début de dégradation (Tab. VI).

Tableau VI. Températures de dégradation des polymères étudiés.

\begin{tabular}{lc}
\hline Échantillon & Température de dégradation $\left( \pm 3{ }^{\circ} \mathrm{C}\right)$ \\
\hline BIOMET® & 260 \\
Résine acrylique A & 190 \\
Colophane & 150 \\
Résine acrylique A + colophane & 150 \\
DERAKANE® & 205 \\
Résine acrylique B & 150 \\
Résine acrylique C & 150 \\
\hline
\end{tabular}

La température de début de dégradation est la température pour laquelle la perte de masse atteint $1 \%$ par minute. Tous les échantillons ont une température de dégradation supérieure ou égale à $150{ }^{\circ} \mathrm{C}\left( \pm 3{ }^{\circ} \mathrm{C}\right)$. La dégradation des différents polymères est brutale dès que la température de début de dégradation est franchie. La température de $140{ }^{\circ} \mathrm{C}$ a été choisie pour réaliser l'ensemble des expériences de façon à respecter l'intégrité des matériaux.

Les différentes plaques échantillons ont été placées en immersion, à température ambiante, pendant un mois. Des prélèvements réguliers ont été réalisés et immédiatement analysés par coulométrie. Les résultats obtenus sont rassemblés dans le tableau VII. 
Tableau VII. En gras : teneur massique en eau des films de polymères au cours de leur immersion, \% en masse, (en italique) : écart-type sur les cinq mesures réalisées.

Temps BIOMET ${ }^{\circledast}$ Résine A Résine A+ Résine B Résine C Derakane ${ }^{\circledR}$ (jours)

colophane

\begin{tabular}{lcccccc}
\hline 0 & $\mathbf{0 , 3 6 5}$ & $\mathbf{0 , 2 2 2}$ & $\mathbf{0 , 5 1 2}$ & $\mathbf{0 , 6 0 1}$ & $\mathbf{0 , 3 1 2}$ & $\mathbf{0 , 2 4 0}$ \\
& $(0,036)$ & $(0,017)$ & $(0,019)$ & $(0,067)$ & $(0,021)$ & $(0,025)$ \\
4 & $\mathbf{0 , 4 5 7}$ & $\mathbf{0 , 2 4 3}$ & $\mathbf{1 , 1 1}$ & $\mathbf{0 , 8 9 0}$ & $\mathbf{1 5 , 8}$ & $\mathbf{0 , 3 1 2}$ \\
& $(0,024)$ & $(0,023)$ & $(0,10)$ & $(0,078)$ & $(0,5)$ & $(0,023)$ \\
8 & $\mathbf{0 , 8 6 6}$ & $\mathbf{0 , 3 9 1}$ & $\mathbf{1 , 8 5}$ & $\mathbf{1 , 5 0}$ & $\mathbf{3 0 , 4}$ & $\mathbf{0 , 4 3 2}$ \\
& $(0,035)$ & $(0,016)$ & $(0,17)$ & $(0,15)$ & $(1,1)$ & $0,039)$ \\
10 & $\mathbf{0 , 8 9 0}$ & $\mathbf{0 , 4 3 2}$ & $\mathbf{1 , 9 0}$ & $\mathbf{1 , 5 3}$ & $\mathbf{4 2 , 7}$ & $\mathbf{0 , 5 6 4}$ \\
& $(0,096)$ & $(0,015)$ & $(0,13)$ & $(0,13)$ & $(3,3)$ & $(0,054)$ \\
15 & $\mathbf{0 , 9 1 5}$ & $\mathbf{0 , 5 6 7}$ & $\mathbf{1 , 9 5}$ & $\mathbf{1 , 5 6}$ & $\mathbf{7 7 , 5}$ & $\mathbf{0 , 6 5 6}$ \\
& $(0,112)$ & $(0,022)$ & $(0,08)$ & $(0,06)$ & $(1,2)$ & $(0,083)$ \\
17 & $\mathbf{0 , 9 5 4}$ & $\mathbf{0 , 6 1 2}$ & $\mathbf{1 , 9 7}$ & $\mathbf{1 , 6 1}$ & $*$ & $\mathbf{0 , 6 6 0}$ \\
& $(0,102)$ & $(0,031)$ & $(0,11)$ & $(0,14)$ & & $(0,067)$ \\
21 & $\mathbf{1 , 0 4}$ & $\mathbf{0 , 6 2 1}$ & $\mathbf{2 , 0 2}$ & $\mathbf{1 , 6 2}$ & $*$ & $\mathbf{0 , 6 7 2}$ \\
& $(0,11)$ & $(0,037)$ & $(0,20)$ & $(0,19)$ & & $(0,047)$ \\
25 & $\mathbf{1 , 0 5}$ & $\mathbf{0 , 6 5 4}$ & $\mathbf{2 , 0 3}$ & $\mathbf{1 , 7 1}$ & $*$ & $\mathbf{0 , 6 9 8}$ \\
& $(0,111)$ & $(0,048)$ & $(0,17)$ & $(0,10)$ & & $(0,056)$ \\
30 & $\mathbf{1 , 0 6}$ & $\mathbf{0 , 6 8 1}$ & $\mathbf{2 , 0 3}$ & $\mathbf{1 , 7 9}$ & $*$ & $\mathbf{0 , 7 0 1}$ \\
& $(0,10)$ & $(0,064)$ & $(0,08)$ & $(0,04)$ & & $(0,035)$ \\
& &
\end{tabular}

* : décollement du film, mesure impossible.

Le film à base de résine $\mathrm{C}$ (terpolymère du type MAMAM-BEMA) présente une mauvaise tenue sur le support. Après 15 jours d'immersion, le film se décolle rendant impossible toute analyse ultérieure.

Les écarts-type calculés sont relativement faibles ce qui atteste de la validité du mode opératoire mis en place. La technique de coulométrie permet de quantifier des teneurs en eau très variables allant de valeurs inférieures à $0,2 \%$ et supérieures à $77 \%$. Simple de mise en œuvre, elle permet de comparer le comportement de matériaux très différents.

Les courbes représentant l'évolution de la teneur en eau des films de polymères au cours de leur immersion permet de mieux visualiser les évolutions observées (cf. Fig. 6a).

Parmi les six résines étudiées, un polymère se caractérise par une absorption d'eau très importante. La résine $\mathrm{C}$ absorbe rapidement de l'eau : le film contient plus de $77 \%$ en masse d'eau après 15 jours d'immersion. Cette pénétration d'eau massive peut expliquer le problème de décollement du film du support. La présence des unités « hydrolysables » peut conduire à une dégradation de la matrice. Cette hydrolyse a été mise en évidence et quantifiée par analyse des eaux de vieillissement par chromatographie en phase gazeuse couplée à la spectrométrie de masse (GC-MS). La figure $6 \mathrm{~b}$ présente le suivi de l'hydrolyse au cours des quinze premiers jours d'immersion.

Le pourcentage de groupements hydrolysés est calculé en supposant que tous les groupements « hydrolysables » sont accessibles et réactifs vis-à-vis des molécules d'eau. Les résultats obtenus montrent que la pénétration d'eau mise en évidence par coulométrie conduit à une hydrolyse progressive de la matrice : $20 \%$ des groupements sont hydrolysés après 15 jours d'immersion.
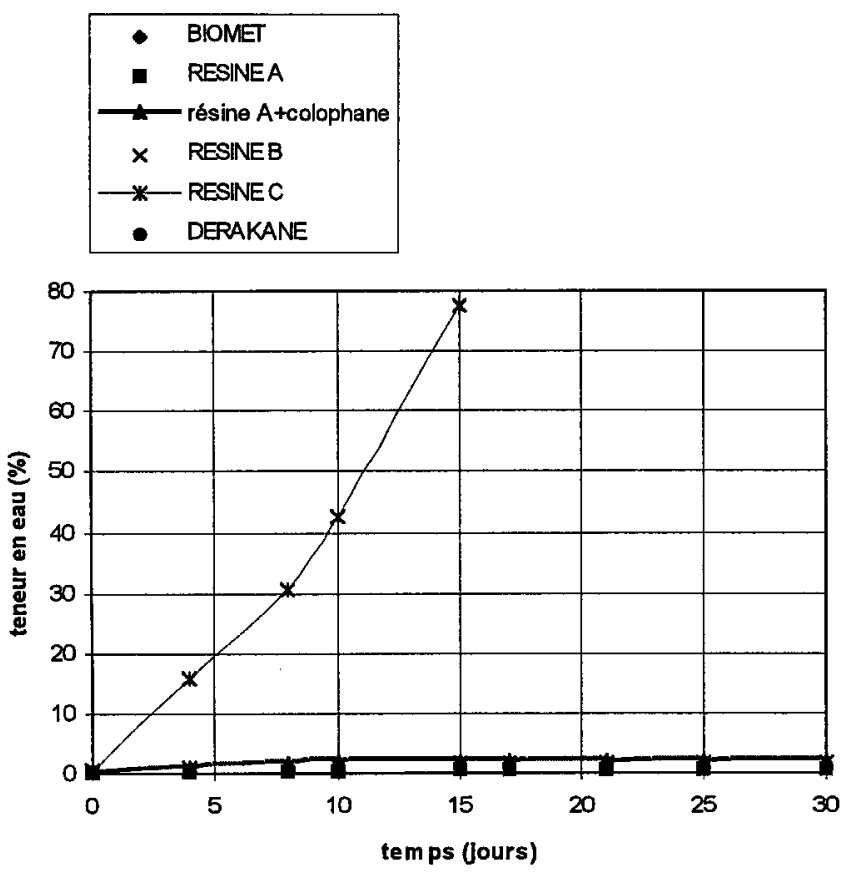

Fig. 6a. Variation de la teneur en eau des films de polymères au cours de l'immersion.

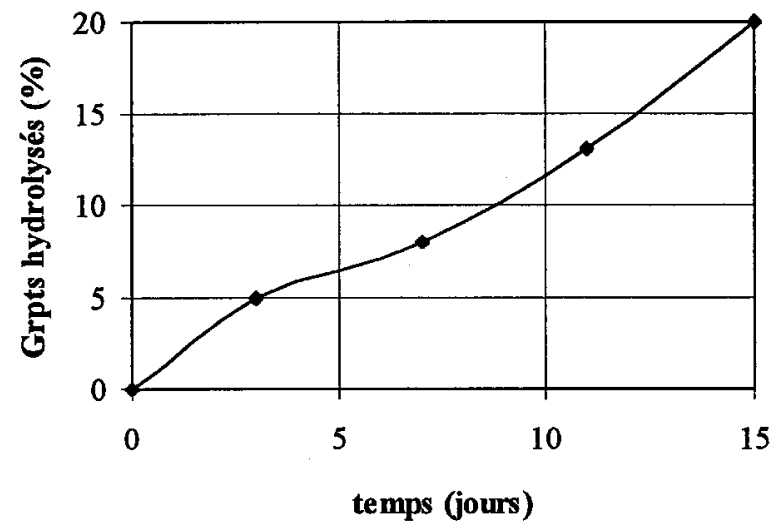

Fig. 6b. Suivi de la dégradation hydrolytique du polymère C par GC-MS.

Les cinq autres résines possèdent un caractère hydrophobe plus marqué (cf. Fig. 7).

Le polymère le plus hydrophobe est la résine acrylique $\mathrm{A}$ (homopolymère du type méthacrylate de méthyle) : la teneur massique en eau est de $0,6 \%$ après 30 jours d'immersion. L'hydrophobie de ce polymère peut être expliquée par la présence des groupements méthyle sur les chaînes latérales. Toutefois, lorsque ce polymère est mélangé avec de la colophane, composé hydrosoluble, il absorbe davantage d'eau ( $1,7 \%$ après un mois d'immersion). Cette comparaison permet de mettre en avant le caractère hydrophile de la colophane qui favorise la pénétration de l'eau dans la matrice polymère.

La DERAKANE ${ }^{\circledR}$, matériau hydrophobe de référence, absorbe légèrement plus d'eau que la résine $\mathrm{A}$ : la teneur massique en eau est de $0,7 \%$ après la même durée. Ce caractère hydrophobe est expliqué par la présence de nombreux cycles aromatiques et de fonctions méthyle dans la structure chimique de cette résine. 


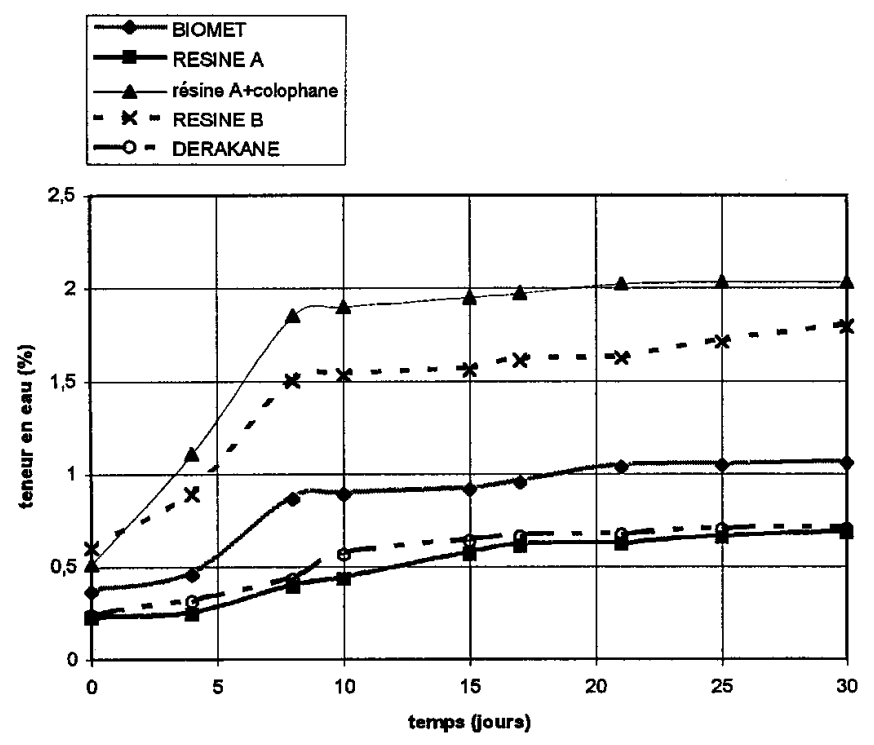

Fig. 7. Variation de la teneur en eau des films de polymères au cours de l'immersion (agrandissement de la Fig. 6a).

Le BIOMET ${ }^{\circledR}$ conserve des teneurs relativement faibles qui se stabilisent autour de $1 \%$ après 15 jours d'immersion. Le caractère hydrophobe du Biomet peut s'expliquer par sa structure chimique originelle qui ne possède que des fonctions hydrophobes (par exemple, méthyle).

La résine $\mathrm{B}$ (copolymère du type MAM-AM) contient des pôles hydrophiles (acide méthacrylique). Elle absorbe rapidement de l'eau au début de l'immersion. Puis la teneur se stabilise autour de 1,6\% après une semaine d'immersion. Ce caractère relativement hydrophobe est inattendu en raison de la présence des groupements $\mathrm{COOH}$ de l'acide. Il peut être expliqué par la présence d'une structure particulière masquant ces groupements hydrophiles. Une orientation des chaînes polymères au cours du séchage du film a été envisagée. Toutefois, sa mise en évidence par spectrométrie de photoélectrons sous bombardement de rayons X (ESCA) n'a pas été possible. Les analyses de surface et « à cœur»du vernis ont conduit à des compositions chimiques semblables (cf. Tab. VIII).

Tableau VIII. Composition chimique en surface et « à cœur» du vernis $\mathrm{B}$.

\begin{tabular}{lcc}
\hline Composition & $C(\%)$ & $O(\%)$ \\
\hline en surface & 80 & 82 \\
«à cœur» & 20 & 18 \\
\hline
\end{tabular}

Ces résultats peuvent s'expliquer par une mauvaise mâ̂trise de la profondeur d'érosion mécanique.

Comparativement à la résine $\mathrm{A}$, le copolymère $\mathrm{B}$ possède un caractère " hydrophobe » marqué $(1,6 \%$ d'eau absorbée après une semaine d'immersion). Ces deux résines de structures chimiques proches (à base de monomères du type MAM et AM) se différencient par la présence de groupe- ments «hydrolysables» dans le cas de la résine A. L'hydrolyse obtenue semble favoriser la pénétration de l'eau dans la matrice (environ $30 \%$ d'eau absorbée après une semaine d'immersion) grâce à une désorganisation des chaînes polymères facilitant l'accès des molécules d'eau.

Les résultats obtenus confirment qu'un liant acrylique hydrophobe (poly MAM, par exemple) absorbe de très faibles quantités d'eau au cours d'une immersion à température ambiante. Toutefois, dans le cadre de l'application envisagée, une pénétration d'eau suffisante doit être obtenue pour permettre la libération des charges toxiques et la protection antisalissures. Il apparaît donc nécessaire d'incorporer des unités hydrophiles par mélange (mélange de la résine A et de la colophane) ou par copolymérisation (résine B). D'autre part, les résultats obtenus montrent que la pénétration de l'eau dans la matrice devient très importante lorsqu'elle s'accompagne d'une dégradation hydrolytique (résine C). L'hydrolyse permet une dégradation progressive de la matrice et conduit à la désorganisation des chaînes polymères facilitant la pénétration de l'eau.

Parallèlement à cette étude de la perméabilité des films de vernis, les résines ont été évaluées comme liant pour peinture antisalissures. Parmi les résines acryliques $\mathrm{A}, \mathrm{B}$ et $\mathrm{C}$, seule la résine $\mathrm{C}$ (terpolymère du type MAM-AMBEMA) a conduit à une peinture présentant une action antisalissures importante sur une période de deux ans. Cette résine est conservée pour la suite de l'étude concernant l'effet des charges.

\section{Vernis chargés}

Les différentes plaques échantillons réalisées à base de résine $\mathrm{C}$ ont été placées en immersion pendant un mois. Des prélèvements réguliers ont été réalisés et immédiatement analysés par coulométrie. Les résultats obtenus sont représentés sur la figure 8 :
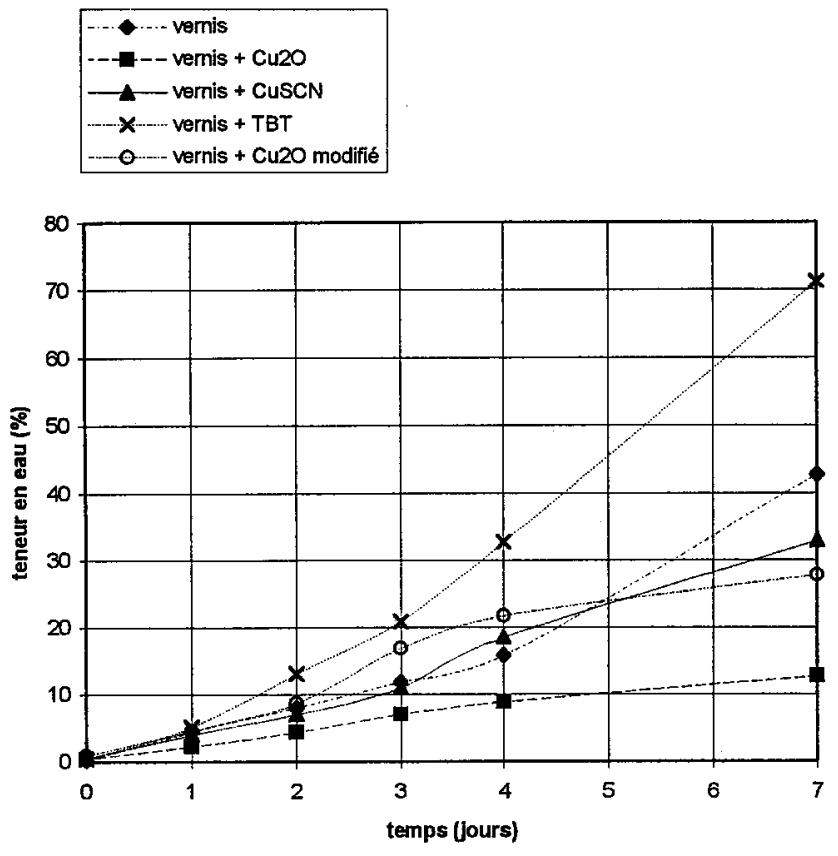

Fig. 8. Teneur en eau des films de résines $\mathrm{C}$ comportant chacun une charge différente. 
Les charges modifient nettement le comportement de la résine C vis-à-vis de l'eau. L'oxyde de cuivre joue un rôle régulateur : sept jours après l'immersion, la teneur en eau n'est que de $12 \%$. L'ajout d'oxyde de cuivre masque partiellement le caractère hydrophile du liant. Toutefois, cet effet disparaît lorsque l'oxyde de cuivre est enrobé par une substance polymérique : la teneur en eau atteint $28 \%$ après sept jours d'immersion. Cette observation illustre l'effet des traitements de surface appliqués aux pigments et aux charges sur les propriétés finales des revêtements. L'effet « hydrophobe » de l'oxyde de cuivre peut s'expliquer par l'établissement d'interactions spécifiques entre les atomes de cuivre et d'oxygène. Le biocide $\mathrm{CuSCN}$ semble légèrement hydrophobe mais ne modifie pas le comportement du liant vis-àvis de l'eau de façon significative. Le TBT favorise nettement la pénétration d'eau et exacerbe le caractère hydrophile de la résine $\mathrm{C}$ : la teneur en eau dépasse $70 \%$ après sept jours d'immersion. Ceci peut s'expliquer par le caractère hydrophile du sel : $\mathrm{Sn}^{+}(\mathrm{Bu})_{3}, \mathrm{~F}^{-}$.

\section{Conclusion}

La coulométrie est une technique simple mais puissante pour étudier la pénétration de l'eau dans des films de polymères chargés ou non. Elle permet de quantifier précisément de faibles teneurs (de l'ordre de 0,2\% massique) mais également des teneurs très élevées (supérieures à $77 \%$ massiques). Le protocole de prélèvement proposé se trouve validé par les faibles écarts-type obtenus sur les cinq mesures réalisées pour chacun des échantillons. La coulométrie nous a permis d'apprécier de façon plus fiable le caractère «hydrophobe/hydrophile» des liants et des charges utilisés dans les peintures antisalissures. La méthode proposée permet une évaluation rapide du comportement du liant et évite de nombreux essais de formulation.

Peu utilisée jusqu'à présent dans le domaine des peintures antisalissures, la coulométrie semble être une méthode de choix pour étudier l'évolution de revêtements en atmosphère humide et optimiser les formulations. Cette méthode n'est pas limitée à la caractérisation des peintures. D'autres applications peuvent être envisagées : dosage de l'eau présente dans des films de polymères utilisés dans les domaines de l'agriculture pour le relargage d'engrais, insecticides et pesticides (à effet retard) et de la pharmacie (libération contrôlée de principes actifs).

\section{Références}

1. Henry, C. M. J. Coat. Tech. 1990, 62, 33.

2. Stratmann, M.; Hoffmann, K. Proc. 9th European Cong. on Corrosion, Utrecht, The Netherlands, 1989; p. CO-70.

3. Van Westing, E. P. M.; Ferrari, G. M.; De Wit, J. H. W. Corros. Sci. 1994, 36, 957.

4. Alfrey, T.; Gurnee, E. F.; Lloyd, W. G. J. Polym. Sci., part C 1966, 12, 249.

5. Braesher, D. M.; Kinsbury, A. H. J. Appl. Chem. 1954, 4, 62.

6. Gimenz, P.; Petit, D.; Badia, M. Mater. Sci. Forum 1986, 8, 315.

7. Leidheiser, H.; Kendig, M. Corrosion 1976, 32(2), 69.

8. Salisburg, M. K.; Clargue, A. D. H.; Herbert, I. R.; Cotgreave, T.; Mansfield, P.; Taylor, D. Second Inter. Conf. on Polymers in a Marine Environnement, London 1989, Marine Management Holdings Ltd, p. 131.

9. Meares, P. Second Inter. Conf. on Polymers in a Marine Environnement, London 1989, Marine Management Holdings Ltd, p. 149.

10. Laoubi, S.; Vergnaud, J. M. Eur. Polym. J. 1991, 27 (12), 1425.

11. Steinsmo, U. "Evaluation of corrosion protective organic coatings for steel structures in fresh water", rapport, Sel. Ind. Tek. For., 1993.

12. Casanova, J. C. «Caractérisation de la dégradation en milieu marin d'un revêtement époxy-vinylique d'anticor rosion déposé sur un substrat d'acier », thèse de doctorat, Université de Marseille, 1994.

13. Linquist, S. A. Corrosion 1983, 41, 69. 